\title{
Diseño de un proyecto para implementar la simulación como metodología didáctica en el Grado de Enfermería
}

\author{
Martínez Isasi, Santiago; Sobrido Prieto, Natalia; Sobrido Prieto, María
}

Facultad Enfermería y Podología. Universidade da Coruña

\section{RESUMEN}

La simulación se ha ido introduciendo en los procesos educativos en el ámbito sanitario, siendo un pilar en la metodología docente en nuestros días. La simulación se define como las actividades que imitan la realidad del entorno clínico, diseñado para entrenar procedimientos, toma de decisiones y aplicar el pensamiento crítico. Mediante la simulación se trabajan habilidades técnicas y habilidades no técnicas basadas en el CRM (Crew Resource Management) y en la cultura de seguridad y hacen referencia, entre otras, al trabajo en equipo, el liderazgo y a la comunicación eficaz.

El objetivo principal del proyecto es introducir la simulación clínica en el Grado de Enfermería.

El proyecto consta de 3 fases diferenciadas. La primera fase es la creación de un grupo de trabajo de profesorado voluntario en el cual se trabajarán varios puntos: estructura, dotación económica y objetivos; la segunda fase se realizará la propuesta del proyecto; la tercera fase será la formación del profesorado.

La implementación de la simulación en el Grado de Enfermería será secuencial y se realizarán una evaluación anual y final, por alumnos y docentes del Grado de Enfermería.

PALABRAS CLAVE: Enfermería, simulación, educación 


\section{CITA RECOMENDADA:}

Martínez Isasi, S., Sobrido Prieto, N., Sobrido Prieto, M. (2018). Diseño de un proyecto para implementar la simulación como metodología didáctica en el Grado de Enfermería. En E. de la Torre Fernández (ed.) (2018). Contextos universitarios transformadores: retos e ideas innovadoras. II Xornadas de Innovación Docente. Cufie. Universidade da Coruña (pág. 351-360).

DOI capítulo: https://doi.org/10.17979/spudc.9788497496780.351

DOl libro: https://doi.org/10.17979/spudc. 9788497496780

\section{ABSTRACT}

Simulation has been introduced into educational processes in healthcare, being a pillar in the teaching methodology in nowdays. Simulation is described as the activities that mimic the reality of the clinical setting, designed to train procedures, decision making and applying critical thinking. Simulation assist technical and non-technical skills based on the CRM (Crew Resource Management) and safety culture and which make reference to teamwork, leadership and effective communication.

The main objective of the project is to introduce clinical simulation in the Nursing Degree.

The project consists of 3 stages. The first stage will be the creation of a working group of voluntary teachers in which several points will work on: structure, financial resources and objectives; the second stage will be present the project proposal; the third stage will be teacher training. The implementation of the simulation in the Nursing Degree will be sequential and there will be an annual and final evaluation by students and teachers of the Nursing Degree.

KEY WORDS: Nursing, simulation, educational 


\section{INTRODUCCIÓN}

La simulación que hoy conocemos, nació como concepto moderno en 1929 con la presentación del primer simulador de vuelo llamado "Link Trainer", desarrollado por Edwin A. Link que ofreció una nueva e innovadora alternativa para el entrenamiento de pilotos de guerra (Reznek, Harter \& Krummel, 2002). Este tipo de entrenamiento enfatiza la repetición de situaciones y manejo de eventos para mejorar la retención y el aprendizaje, a la vez que favorece la reflexión activa y el análisis como una potente herramienta pedagógica. Desde ese momento ha ido introduciéndose de manera progresiva en los procesos educativos en el ámbito sanitario, siendo muy importante como metodología docente en nuestros días.

La simulación puede tomar muchas formas, desde relativamente simple a muy compleja. La fidelidad se define, en el contexto de la simulación, como el "grado en que una simulación reproduce la realidad"; las dimensiones básicas de la fidelidad son la física, psicológica y conceptual. Resulta evidente que el grado de fidelidad está estrechamente relacionado con el tipo de simulador que se emplea; máxime cuando los actuales avances tecnológicos permiten un grado de realismo (tanto en el equipo como en la representación psicológica y conceptual) muy elevados (International Nursing Association for Clinical Simulation and Learning, 2011).

Las posibilidades de la simulación como herramienta docente están ampliamente demostradas, empleándose a lo largo de los años para el aprendizaje de habilidades técnicas. En los últimos años, al aumentar la tecnología de los simuladores se han ampliado las posibilidades de aprendizaje, pero ello no debe alterar el orden normal del aprendizaje, empezando por simulación de baja fidelidad para entrenar habilidades genéricas y simuladores de mayor fidelidad para entrenar a profesionales 0 alumnos con experiencia y conocimientos adecuados.

La metodología de simulación empleada en ciencias de la salud fue descrita por Alinier (2007) y Alinier (2011) donde describe las herramientas y las metodologías que se utilizan en simulación. 
En la actualidad, la simulación clínica se define como las actividades que imitan la realidad del entorno clínico, diseñado para entrenar procedimientos, toma de decisiones y aplicar el pensamiento crítico (Chisari et al. 2005); abarcando técnicas tales como juegos de rol y el uso de videos interactivos o maniquíes.

La simulación como herramienta pedagógica participativa emplea el modelo de Miller (1990) en que establece una evaluación del aprendizaje por competencias.

Ilustración 1. Pirámide de Miller

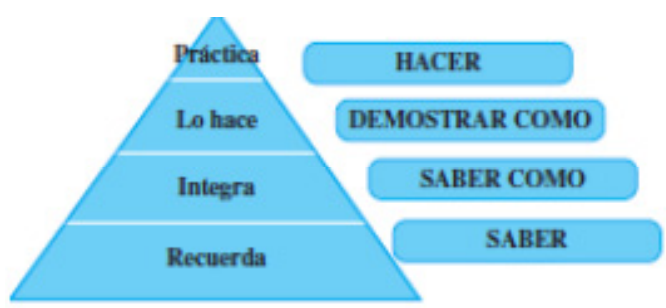

En la simulación se pueden trabajar habilidades técnicas (Vargas, 2007) y habilidades no técnicas (Flin, O'Connor, \& Crichton 2008) basadas en el CRM (Crew Resource Management) y en la cultura de seguridad, ya que favorece la seguridad de los pacientes y evita el error y se trabajan habilidades como el trabajo en equipo, el liderazgo y la comunicación eficaz (Rall, Glavin \& Flin, 2008), (Rall, \& Dieckmann, 2005) (Rall, \& Dieckmann, 2005).

Tabla 1. Puntos del CRM

\begin{tabular}{|c|c|c|c|c|c|}
\hline 1 & Conocer el entorno & 6 & $\begin{array}{l}\text { Movilizar todos } \\
\text { recursos disponibles }\end{array}$ & 11 & $\begin{array}{l}\text { Utilizar } \\
\text { cognitivas }\end{array}$ \\
\hline 2 & Anticipar y planificar & 7 & Comunicarse eficientemente & 12 & $\begin{array}{l}\text { Re-evaluar } \\
\text { periódicamente }\end{array}$ \\
\hline 3 & Pedir ayuda pronto & 8 & $\begin{array}{l}\text { Utilizar toda la información } \\
\text { disponible }\end{array}$ & 13 & Buen trabajo de equipo \\
\hline 4 & $\begin{array}{l}\text { Ejercer el liderazgo y } \\
\text { saber seguirlo }\end{array}$ & 9 & $\begin{array}{l}\text { Prevenir y manejar los } \\
\text { errores de fijación }\end{array}$ & 14 & $\begin{array}{l}\text { Repartir la atención de } \\
\text { forma juiciosa }\end{array}$ \\
\hline 5 & $\begin{array}{l}\text { Distribuir la carga de } \\
\text { trabajo }\end{array}$ & 10 & $\begin{array}{l}\text { Comprobaciones cruzadas } \\
\text { (dobles) }\end{array}$ & 15 & $\begin{array}{l}\text { Establecer prioridades de } \\
\text { forma dinámica }\end{array}$ \\
\hline
\end{tabular}


La simulación en el Grado de Enfermería se ha realizado históricamente, pero sin llegar a completar el círculo de la enseñanza, sin trabajar las habilidades técnicas y no técnicas de manera conjunta y sin aprendizaje reflexivo final. Mediante la simulación se pueden trabajar la mayoría de las habilidades que practica un alumno de enfermería y los escenarios a los que se enfrentará durante su ejercicio profesional.

\section{OBJETIVOS}

Los objetivos generales son:

- Introducir la simulación como herramienta docente en el Grado de Enfermería.

- Adecuar la metodología de la formación práctica en el Grado de Enfermería a la simulación.

- Fomentar una formación integral basada en el entrenamiento de habilidades técnicas y no técnicas con un aprendizaje reflexivo.

Y objetivos específicos:

- Conseguir que el alumno se involucre de manera activa en el proceso de aprendizaje.

- Aumentar la calidad de la formación del Grado de Enfermería.

- Evaluar las competencias que adquieren los alumnos durante sus estudios.

\section{DESARROLLO DEL PROYECTO}

Para poder llevar a cabo este proyecto, se considerarán 3 fases secuenciales.

- Fase inicial: se creará un grupo de trabajo de profesorado voluntario, encargados de llevar el peso del proyecto. En un primer momento, el grupo de trabajo identificará necesidades y potenciales problemas y realizará un inventario del material disponible. El resultado de este informe se remitirá a los coordinadores de cada curso, estancias clínicas y coordinador de grado.

- Fase 2: se realizará la propuesta del proyecto en la que: a) se definirá la idea del proyecto, b) se expondrán los objetivos, c) se definirá de manera precisa la 
incorporación de la simulación a los programas docentes, la modificación de los planes de organización docente y se creará un mapa curricular, d) se realizará la provisión de fondos inicial y de mantenimiento y e) se establecerá un plan de seguimiento, mediante una subcomisión de simulación en la comisión de docencia.

- $\quad$ Fase 3: será la formación del profesorado. Para ello se explorarán las necesidades y fortalezas de los profesores del departamento y se establecerá un plan formativo en el cual ellos mismo serán docentes y alumnos.

\section{IMPLANTACIÓN}

La simulación se incorporará paulatinamente en el Grado de Enfermería. El primer año se comenzará con $1^{0}$ y se aumentará un curso cada año. Al finalizar cada año se realizará una evaluación de las materias y programas docentes para poder ser revisadas y modificadas. Una vez finalizado el primer ciclo de alumnos que completen el grado con la simulación en su programa docente se realizará una evaluación completa y final.

Una vez realizada las diferentes evaluaciones se reunirá a subcomisión de simulación y analizará los datos para poner en marcha planes de mejora si fuese necesario.

En base a experiencias similares y opiniones de expertos podemos prever/suponer los beneficios, ventajas, obstáculos e inconvenientes a los que nos vamos a enfrentar.

La simulación tiene grandes beneficios en el proceso de la enseñanza:

- Para el alumnado: la simulación aporta un mayor control de su aprendizaje al ser parte activa y provoca un entusiasmo en todo el proceso, especialmente en el proceso de análisis reflexivo. Los alumnos aprenden practicando en situaciones simuladas, aumentado su confianza y seguridad en el momento que se enfrenten a un paciente

- Para el profesorado: facilita la evaluación de competencias generales, tal y como se indica en el Plan Bolonia, hace partícipe al alumno de su proceso de aprendizaje y establece un feedback continuo alumno-profesor. 
- Para la Institución: obtendrá una valoración positiva al presentar títulos de grado con una metodología actual, innovadora y estandarizada.

La implantación de la simulación en el Grado en Enfermería de la Facultad de Enfermería y Podología supone una gran oportunidad si tenemos en cuenta los siguientes aspectos:

La presencia en el Grado de Enfermería de profesores asociados con un perfil clínico, que dotará a los escenarios de un mayor realismo y cercanía con la práctica asistencial.

La posibilidad de distribución de alumnos en grupos pequeños, máximo de 12, que permitirá optimizar al máximo la herramienta docente.

La simulación es un campo con mucho potencial investigador en salud, ya que trabaja aspectos de relevancia sanitaria como son la seguridad, la comunicación entre profesionales y pacientes, el trabajo en equipo y el liderazgo entre otros. Al personal docente e investigador le abre una línea de investigación cualitativa y cuantitativa.

Otra ventaja, será el reto docente al que se enfrentarán los docentes, ya que aunque la simulación lleva implantada en los estudios de enfermería muchos años, no se han explorado todas las fases del círculo de la enseñanza con un aprendizaje reflexivo final. La curva de aprendizaje se ve acortada, se estimula la autoevaluación y el refuerzo positivo (a través de la grabación y el debreafing). La simulación se realiza en un ambiente seguro para el paciente y el alumno, en el que errar está permitido y será la base del aprendizaje.

Los docentes y/o investigadores se encontrarán ante un reto por la aplicación de una metodología innovadora en la que el principal beneficiado es el alumno.

Las principales barreras que el proyecto se podría encontrar son:

- En los alumnos: se puede ver sometido a una presión añadida en la simulación, que puede limitar los beneficios de la simulación

- Al PDI: le supondrá una carga de trabajo superior y un cambio en la metodología que venía empleando, con lo que podría presentarse una resistencia al cambio. 
- En la Institución: la simulación lleva implícita una inversión y un coste de mantenimiento. Supondrá un esfuerzo importante en buscar fuentes de financiación alternativas.

Se necesita formar a los profesores en este tipo de metodología que en muchos casos es difícil de asumir.

Se debe restructurar el plan de organización docente al realizar las simulaciones en grupos reducidos y, en muchos casos, debe ser realizado por 2 docentes.

\section{PLAN DE TRABAJO}

De acuerdo al plan a las fases previamente establecidas, este proyecto lo podremos dividir en dos fases claramente diferenciadas; a) desarrollo del proyecto y b) implantación en el grado

llustración 2: desarrollo del proyecto

\begin{tabular}{|l|l|l|l|}
\hline & Enero-Febrero 2018 & Marzo-Abril 2018 & Junio-Julio 2018 \\
\hline Fase 1 & & & \\
\hline Fase 2 & & & \\
\hline Fase 3 & & & \\
\hline
\end{tabular}

Ilustración 3: Implantación en el grado

\begin{tabular}{|l|l|l|l|l|}
\hline & Curso 2019-20 & Curso 2020-21 & Curso 2021-22 & Curso 2022-23 \\
\hline $1^{0}$ curso & & & & \\
\hline $2^{0}$ curso & & & & \\
\hline $3^{0}$ curso & & & & \\
\hline $4^{0}$ curso & & & & \\
\hline
\end{tabular}




\section{REFERENCIAS}

- Alinier, G. (2007). A typology of educationally focused medical simualtion tools. Medical Teacher, 29: e243-e250.

- Alinier, G. (2011). Developing high-fidelity health care simulation scenarios: a guide for educators and professional. Simulation and Gaming, 42:9.

- Chisari, G. Brown, C. Calkins, M. Echthernacht, M. Kearney-Nunnery, R. Knopp, B. Spector, N. (2005). Clinical instruction in prelicensure nursing program. National Council of State Boards of Nursing (NCSBN).

- Flin, R., O'Connor, P., \& Crichton, M. (2008). Safety at the sharp end: A guide to nontecnical skills. Hamphshire, England: Ashgate Publishing Companys

- International Nursing Association for Clinical Simulation and Learning, I. (2011). The debriefing process. Clinical Simulation in Nursing, 7,s16-s17.

- Miller, G.E. (1990). The assessment of Clinical Skills/ Competence/ Performance. Academic Medicine, 65 (9):63-7.

- Reznek, M. Harter, P. Krummel, T. (2002).Virtual reality and simulation: training the future emergency physician. Acad Emerg Med, 9: 78-87.

- Rall, M., \& Dieckmann, P. (2005). Crisis Resource Management to improve patient safety. Viena, Austria: European Society of anaesthesiology.

- Rall, M., \& Dieckmann, P. (2005). Simulation and patient safety: The use of simulation to enhance patient safety on a systems level. Current anaesthesia and Critical Care, 16(5),273-281.

- Rall, M., Glavin, R., \& Flin, R. (2008). The 10 seconds for 10 minutes principle. Why things go wrong and stopping them getting worse. Bulletin of The Royal College of anaesthetist Special human factors, 2614-2616.

- Vargas, F. Key (2007). Competencies and Lifelong Learning. Montevideo: Cinterfor. 